\title{
Huanglian-Jie-Du-Tang Extract Ameliorates Depression-Like Behaviors through BDNF-TrkB-CREB Pathway in Rats with Chronic Unpredictable Stress
}

\author{
Yi-Lu Ye, ${ }^{1}$ Kai Zhong, ${ }^{1}$ Dan-Dan Liu, ${ }^{1}$ Jing Xu, ${ }^{1}$ Bei-Bei Pan, ${ }^{2}$ Xiang Li, ${ }^{1}$ \\ Yue-Ping Yu, ${ }^{1}$ and Qi Zhang ${ }^{1}$ \\ ${ }^{1}$ Basic Medicine Department, Hangzhou Medical College, Hangzhou 310053, China \\ ${ }^{2}$ The Second Affiliated Hospital of Zhejiang University School of Medicine, Hangzhou 310009, China \\ Correspondence should be addressed to Qi Zhang; zhangqi@hmc.edu.cn
}

Received 20 January 2017; Revised 19 April 2017; Accepted 10 May 2017; Published 14 June 2017

Academic Editor: Krishnadas Nandakumar

Copyright (c) 2017 Yi-Lu Ye et al. This is an open access article distributed under the Creative Commons Attribution License, which permits unrestricted use, distribution, and reproduction in any medium, provided the original work is properly cited.

\begin{abstract}
Neuroinflammation is considered as one of the common pathogeneses of depression. Huanglian-Jie-Du-Tang (HJDT) is a traditional Chinese herbal formula. The present study investigates the antidepressant-like effect of HJDT and its possible mechanism in rats. Rats were given $\operatorname{HJDT}(2,4$, and $8 \mathrm{~g} / \mathrm{kg}$, intragastrically), paroxetine ( $1.8 \mathrm{mg} / \mathrm{kg}$, intragastrically), or an equivalent volume of saline for 42 days. The depression-related behaviors, including sucrose preference test (SPT), open field test (OFT), novel objective recognition task (NORT), and forced swimming test (FST), were detected. 5-Hydroxytryptamine (5-HT) and dopamine (DA) contents, microglial activation, proinflammatory cytokines, and brain derived neurotrophic factor (BDNF), tropomyosin receptor kinases B (TrkB), and cAMP-responsive element binding protein (CREB) expression were investigated. The results indicated HJDT ( 2 and $4 \mathrm{~g} / \mathrm{kg}$ ) dramatically ameliorated the depression-like behaviors. Also HJDT decreased the number of microglia and the proinflammatory cytokines in hippocampus. Western-blotting analysis displayed HJDT upregulated BDNF, TrkB, and pCREB/CREB expression in hippocampus. Particularly, pCREB DNA activity enhanced with HJDT treatment in hippocampus. But there was no difference in the 5-HT and DA contents with HJDT treatment. In conclusion, it was supposed that HJDT might be a potential Chinese medicine decoction for treating or alleviating complex symptoms of depression through BDNF-TrkB-CREB pathway.
\end{abstract}

\section{Introduction}

Depression is a serious mental disorder with a high prevalence worldwide and causes serious consequences for the individual's quality of life [1]. At present most effective antidepressants to treat depression target serotonin and/or norephinepherine. However, many of these drugs have a slow onset of action (over 2 weeks) and are effective in only 50\% of patients, with serious adverse reactions [2]. Therefore, it is necessary to explore a more powerful and safer antidepressants, which may influence different targets. Traditional Chinese Medicine (TCM) has been commonly considered effective and safe, including many herbal formulae with psychotropic potential.
Huanglian-Jie-Du-Tang (HJDT) is a traditional Chinese herbal formula composed of the roots of Coptis chinensis Franch., Scutellaria baicalensis Georgi, Phellodendron chinense Schneid., and the fruit of Gardenia jasminoides Ellis, which is widely used in China, Japan, and Korea. It was recorded in the traditional Chinese medicinal book WaiTai-Mi-Yao. At present it is widely used for alleviating the symptoms of gastrointestinal issues [3], liver damage [4], Alzheimer's disease [5], ischemic brain injury [6,7], and so forth. In our previous study, the HPLC results demonstrated that the main active components of HJDT were berberine, baicalin, wogonoside, and gardenoside [7], all of which had been found with antidepressant effect. Berberine could attenuate depression- and anxiety-like behaviors following chronic 


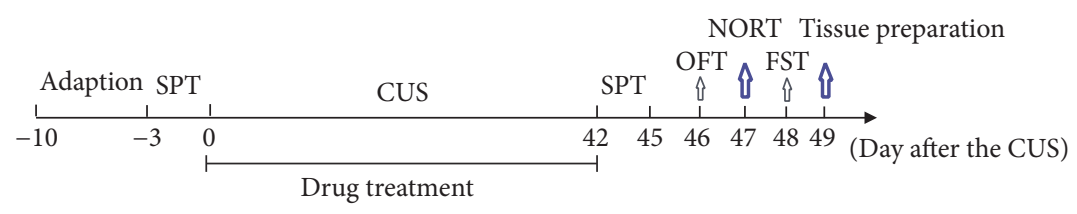

FIGURE 1: Schedule of the experimental protocol.

morphine withdrawal in rats [8]. By normalizing glucocorticoid receptor (GR) function through SGK1- and FKBP5mediated GR phosphorylation, baicalin also improved the depression-like behaviors induced by chronic mild stress in mice [9]. So it was speculated that HJDT could have the basis on anxiolytic and antidepressant effects.

Microglia are important resident immunoreactive cells in the central nervous system (CNS). Commonly, microglia were considered to be "resting," becoming "activated" upon allostatic changes to coordinate immune-like responses [10] and triggering inflammation reactions through chemokine and cytokine [11]. The available evidence indicates that depression is tightly associated with alterations of microglia and inflammation [12], which manifested with more proinflammatory profile [13] and decreased microglia-derived brain derived neurotrophic factor (BDNF) [14]. BDNF and its receptor tropomyosin receptor kinases $\mathrm{B}(\operatorname{TrkB})$ as well as its downstream target cAMP-responsive element binding protein (CREB) played crucial role in depression $[15,16]$. Therefore this study examined the effects of HJDT on the depression-like behaviors and potential mechanism involved in inflammatory reaction and BDNF-TrkB-CREB pathway.

\section{Materials and Methods}

2.1. Plant Materials and HJDT Preparation. The roots of Coptis chinensis Franch. (141107, Sichuan, China), Scutellaria baicalensis Georgi (150113, Neimenggu, China), Phellodendron chinense Schneid. (150203, Sichuan, China), and the fruit of Gardenia jasminoides Ellis (140926, Jiangxi, China) were purchased from Eastern China Medical Corp. (Zhejiang Province, China) and identified as being in families Ranunculaceae, Labiatae, Rutaceae, and Rubiaceae, respectively. Then they were authenticated by Professor H. X., Sun (College of Animal Sciences, Zhejiang University). According to our previous report, aqueous extract of HJDT was prepared [7]. The ultima concentration was $1 \mathrm{~g} / \mathrm{mL}$.

2.2. Active Components of HJDT Determination. The HPLC was carried out as described in our previous study [7]. Briefly, HPLC conditions are as follows: eluent A, acetonitrile; eluent $\mathrm{B}, 0.05 \%$ potassium dihydrogen phosphate and $0.05 \%$ triethanolamine-water ( $\mathrm{pH} 3.0$ adjusted by phosphoric acid); gradient, 0 min $(A-B, 14: 86) \rightarrow 12 \min (A-B, 25: 75) \rightarrow$ $27 \min (A-B, 30: 70) \rightarrow 28 \mathrm{~min}(\mathrm{~A}-\mathrm{B}, 14: 86)$; and then it was equilibrated with $\mathrm{A}-\mathrm{B}(14: 86)$ for $12 \mathrm{~min}$ at a flow of $0.8 \mathrm{~mL} / \mathrm{min}$. The contents of the analytics were determined from the corresponding calibration curves.
2.3. Animals. Male Sprague-Dawley rats (Shanghai Slyke Laboratory Animal Limited Corporation, Certificate number SCXK 2012-0002, China) were handled in accordance with the National Institute of Health Guide for the Care and Use of Laboratory Animals and the norms of the Ethics Committee for Animal Research of Hangzhou Medical College. They were housed under a $12: 12 \mathrm{~h}$ light/dark cycle, controlled temperature of $22 \pm 1^{\circ} \mathrm{C}$, and humidity of $50 \pm 10 \%$. Food and water were provided ad libitum.

2.4. Groups and Drug Treatments. After environmental adaption for one week, the rats were assigned to one of the following groups according to their sucrose preference ratio and body weight: normal control group (only saline treatment and no chronic unpredictable stress (CUS)); CUS depression group (saline treatment and CUS); paroxetine group $(1.8 \mathrm{mg} / \mathrm{kg}$, GlaxoSmithKline, England, paroxetine treatment group and CUS); HJDT groups (2, 4, and $8 \mathrm{~g} / \mathrm{kg}$, HJDT treatment and CUS). Rats were given intragastrically either paroxetine, HJDT, or saline for 42 days after the CUS, and at $1 \mathrm{~h}$ before the beginning of the CUS procedure or behavioral tests. The dose of paroxetine and HJDT were chosen based on the previous reports $[7,17]$.

2.5. Experimental Protocol. Except normal control group, rats in other groups were subjected to two stressors on an unpredictable order and at an unpredictable time each day following gavage for 6 weeks. The stresses included food deprivation for $24 \mathrm{~h}$, water deprivation for $24 \mathrm{~h}$, inversion of day/night cycle, forced swimming for $6 \mathrm{~min}, 45^{\circ}$ tilted cage for $7 \mathrm{~h}$, soiled cage bedding overnight, and restraint for $5 \mathrm{~min}$. Rats in normal control group were left undisturbed during the period. The body weight was measured once weekly from day 0 after the CUS. Sucrose preference test (SPT), open field test (OFT), novel objective recognition task (NORT), and forced swimming test (FST) were performed from day 42 to day 48 after the CUS, respectively (see Figure 1).

\subsection{Behavioral Tests}

2.6.1. Sucrose Preference Test (SPT). The SPT was carried out as previous report with slight modification [18]. The volumes of sucrose solution and water consumed were recorded at the 4 th day during SPT. At that day, rats were housed in individual cages and free to access two bottles containing $100 \mathrm{~mL}$ of sucrose solution $(1 \% \mathrm{w} / \mathrm{v})$ and $100 \mathrm{~mL}$ of water, respectively. After $3 \mathrm{~h}$ of exposure, the volumes of sucrose solution and water consumed were recorded. At last, the sucrose consumption ratio was calculated: sucrose solution 
consumption ratio $(\%)=[$ sucrose solution consumption volume/(water consumption volume + sucrose solution consumption volume) $] \times 100$.

2.6.2. Open Field Test (OFT). Each rat was placed gently into the center of a brightly lit open field arena $(80 \times 80 \times$ $40 \mathrm{~cm}$ ). The locomotor tracks were continuously recorded by a video camera for $5 \mathrm{~min}$ and analyzed by a computerized video tracking system (EthoVision XT, Noldus, Netherlands). The following parameters were determined: (a) total traveled distance (m), (b) time in central region (s), (c) traveled central distance ratio, and (d) rearing number. After each test session, the enclosures were thoroughly cleaned with $70 \%$ ethanol solution.

2.6.3. Novel Object Recognition Task (NORT). The task consisted of three phases: $\mathrm{T}_{1}$ phase, interval time, and $\mathrm{T}_{2}$ phase. During habituation, rats were introduced in the empty chamber and left to freely explore it for $10 \mathrm{~min}$. Afterwards, they were put into their home cages for $5 \mathrm{~min}$, during which two identical objects (familiar object: cubic crude wood with $4 \mathrm{~cm}$ diameter) were placed in the chamber oppositely. In the $\mathrm{T}_{1}$ phase, rats explored the objects for $5 \mathrm{~min}$. After a $1.5 \mathrm{~h}$ interval time, rats were placed into the chamber again for $5 \mathrm{~min}$, when one of the familiar objects was replaced by a novel one (novel object: cylindrical crude wood with $4 \mathrm{~cm}$ diameter and height), that is, $\mathrm{T}_{2}$ phase. Tracks were recorded and analyzed by a computerized video analyzer (EthoVision XT, Noldus, Netherlands). The following parameters were analyzed: total exploration time to familiar and novel objects, discrimination ratio $(\mathrm{DR}, \mathrm{DR}=[N /(N+F)] \times 100 \%)$, and discrimination index $(\mathrm{DI}, \mathrm{DI}=[(N-F) /(N+F)] \times 100 \%)$. $N$ is exploration time with the novel object (min); $F$ is exploration time with the familiar object (min).

2.6.4. Forced Swimming Test (FST). One day before proceeding for the test, rats were trained for swimming for $15 \mathrm{~min}$ in a glass cylinder $(60 \mathrm{~cm}$ height $\times 25 \mathrm{~cm}$ diameter $)$ filled with water at $30 \mathrm{~cm}$ height and under temperature $25 \pm 2^{\circ} \mathrm{C}$. At the next day, rats were individually subjected to swimming for 6 min of which initial 2 min was for acclimatization. And the immobility time during the last 4 min was recorded.

2.7. 5-Hydroxytryptamine (5-HT) and Dopamine (DA) Detection. Rats were decapitated and the hippocampus was separated on ice quickly at day 49 after the CUS. Then it was sonicated to obtain tissue homogenates. After removing particulars by centrifugation $\left(2000 \times \mathrm{g}, 4^{\circ} \mathrm{C}, 20 \mathrm{~min}\right)$, assay was immediately detected. 5-HT (number EIA-3325) and DA (number EIA-3236) were measured using respective ELISA system (Shanghai ELISA Biotech Co., Ltd, China) according to the manufacturer's instructions. Finally, optical density was determined (absorbance at $450 \mathrm{~nm}$ ) on a plate reader.

2.8. Brain Cryosections Preparation and Microglia Number Measurement. Rats were anesthetized after the behavioral tests at day 49. To investigate the activation and the number of microglia in the hippocampal CA1 region and DG, immunohistochemical methods were performed with the polyclonal rabbit antibodies against Iba-1 (1:200, Wako, Japan, number 019-19741). The brain cryosections preparation and immunohistochemical methods were based on our previous report (Ye et al., 2016). Normal goat serum was used instead of the primary antibody in the control sections. The number of positive cells in the hippocampal CA1 region and DG was calculated with three different fields.

2.9. Proinflammatory Cytokines Concentration Measurement. Total RNA was extracted from hippocampus using Trizol reagent (Invitrogen Life Technologies, USA). For cDNA synthesis, $1.5 \mu \mathrm{g}$ RNA was reversely transcribed with superscript $^{\mathrm{TM}}$ III reverse transcriptase (Invitrogen Life Technologies, USA). Semiquantitative PCR was performed using primers specific for TNF- $\alpha$, iNOS, IL- $1 \beta$, IL-6, IL-10, and GAPDH. The primers (Shanghai Yingjun Biotechnology Co., Ltd, China) were as follows:
TNF- $\alpha \quad$ F: $5^{\prime}$ CTGGCGTGTTCATCCGTTCT3'
iNOS F: $5^{\prime}$ TTGGAGCGAGTTGTGGATTG $3^{\prime}$
IL- $1 \beta \quad$ F: $5^{\prime}$ CCTCTGACAGGCAACCACTTA $3^{\prime}$
IL-6 F: $5^{\prime}$ TGCCTTCTTGGGACTGATGT3'
IL-10 F: $5^{\prime}$ TTGAACCACCCGGCATCTAC $3^{\prime}$
GAPDH F: 5'GGAAAGCTGTGGCGTGAT3'

\author{
R: 5'GCCACTACTTCAGCGTCTCGT3' \\ R: $5^{\prime}$ TGAGGGCTTGCCTGAGTGA $3^{\prime}$ \\ R: 5'AAGGTGGAAGAATGGGAGTT3'
}
R: $5^{\prime}$ GCACTGGTCCAAATTCAATTC $3^{\prime}$
R: $5^{\prime}$ ATACTGGTCTGTTGTGGGTGGT3'
R: $5^{\prime}$ CCAAGGAGTTGCTCCCGTTA3'

The products of RT-PCR were separated by electrophoresis using $1.2 \%$ agarose gel and stained with ethidium bromide. Densitometric analysis was performed using image analysis software.

2.10. BDNF, TrkB, CREB, and pCREB Expression Measurement. Samples of hippocampus were homogenized on ice in PBS containing $1 \%$ protease inhibitor and $1 \%$ phosphatase inhibitor for Western-blotting. Proteins were obtained by centrifugation at $14000 \mathrm{rpm}$ at $4^{\circ} \mathrm{C}$ for $15 \mathrm{~min}$ and quantified by Bradford assay (BioRad, USA). A $50 \mu \mathrm{g}$ sample of each group was subjected to electrophoresis using 20\% SDS at $80 \mathrm{~V}$. The proteins were transferred to polyvinylidene fluoride membranes at $250 \mathrm{~mA}$ for $2 \mathrm{~h}$. Antibodies for BDNF (1:1000, Santa Cruz Biotechnology, USA, number sc-546), TrkB (1:1000, Santa Cruz Biotechnology, USA, number sc-8316), CREB (1:2000, Cell Signaling Technology, USA, number 9197), pCREB (1:2000, Cell Signaling Technology, USA, number 9198), and GAPDH (1:10000, KangChen BioTech, Shanghai, China) were applied overnight. Then membranes were incubated with horseradish peroxidase (HRP) secondary antibody (KangChen Bio-Tech, Shanghai, China). 
TABLE 1: Contents of active components in the decoction of HJDT (\%) $(\bar{x} \pm \operatorname{SEM}, n=5)$.

\begin{tabular}{lccccccc}
\hline & Geniposide & Baicalin & Palmatine hydrochloride & Berberine hydrochloride & Wogonoside & Baicalein & Wogonin \\
\hline Content (\%) & $1.21 \pm 0.02$ & $5.23 \pm 0.05$ & $0.33 \pm 0.01$ & $4.25 \pm 0.04$ & $1.5 \pm 0.01$ & $0.45 \pm 0.02$ & $0.066 \pm 0.02$ \\
\hline$n$ & 5 & 5 & 5 & 5 & 5 & 5 & 5 \\
\hline
\end{tabular}

At last, the signal intensities of proteins were analyzed using Image J software.

2.11. $p$ CREB DNA Activity Detection. The TransAM assay for pCREB activity (Active Motif, USA, number 42096) was performed according to the manufacturer's instructions. Briefly, oligonucleotides containing the pCREB consensus binding site were immobilized on a 96-well plate. The active forms of pCREB in the nuclear extracts were bound to the oligonucleotides on the plate and detected colorimetrically. The samples were measured at an absorbance of $450 \mathrm{~nm}$ on a spectrophotometer.

2.12. Statistical Analysis. All of the data were expressed as mean \pm SEM. Repeated measures of one-way analysis of variance tests (ANOVA) and Fisher's least significant differences (LSD) post hoc analysis tests were performed to analyze the significance of any treatment effect among the groups by SPPS 16.0 for Windows except the effects of HJDT on NORT. $t$-test was used to compare the difference of DR and $\mathrm{DI}$ between $\mathrm{T}_{2}$ phase and $\mathrm{T}_{1}$ phase, the exploration time to novel and familiar objects. $p<0.05$ was considered as statistical significance.

\section{Results}

3.1. The Content of Active Components in HJDT. Using HPLC, we detected the contents of 7 active components in the decoction of HJDT (Table 1). The results showed the content of baicalin, berberine, wogonoside, and geniposide was higher than others, which was demonstrated as $5.23 \%$, $4.25 \%, 1.5 \%$, and $1.21 \%$, respectively. They may be the main active components of HJDT for depression.

3.2. HJDT Had No Effect on the Body Weight Decrease after the CUS. As shown in Figure 2, all groups were equivalent in their body weight prior to the onset of CUS. The body weight in the normal control group gradually increased from day 7 to day 42 after the CUS. Compared to the normal control group, rats exposed to the CUS showed significantly lower body weight from day 7 to day 42 after the CUS. It showed a significant body weight gain after chronic treatment with paroxetine from day 35 to day 42 after the CUS, whereas HJDT had no effect on it, compared to the CUS depression group.

3.3. HJDT Increased the Sucrose Consumption Ratio in SPT after the CUS. Prior to the onset of CUS, all experimental groups showed similar sucrose consumption ratio (data not shown). But at day 43 after the CUS, CUS depression group showed a significant decrease of sucrose consumption

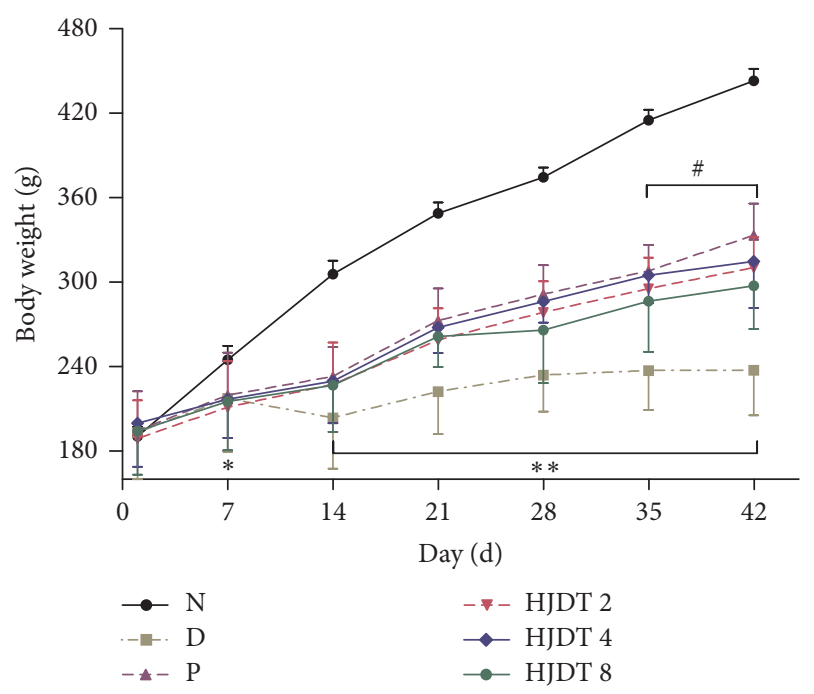

FIgURE 2: Effects of HJDT on the body weight after the CUS in rats. The body weight of rats in CUS depression group decreased from day 7 to day 42 after the CUS. Paroxetine group gained the body weight from day 35 after the CUS. HJDT had no effect on the body weight decrease after the CUS. ${ }^{*} p<0.05$ and ${ }^{* *} p<0.01$ relative to the normal control group; ${ }^{\#} p<0.05$ relative to the CUS depression group. N: normal control group; D: CUS depression group; P: paroxetine group; HJDT 2: HJDT $2 \mathrm{~g} / \mathrm{kg}$ group; HJDT 4: HJDT $4 \mathrm{~g} / \mathrm{kg}$ group; HJDT 8: HJDT $8 \mathrm{~g} / \mathrm{kg}$ group.

ratio, compared with the normal control group (Figure 3). Paroxetine and HJDT ( 2 and $4 \mathrm{~g} / \mathrm{kg}$ ) treatment reversed the decrease of sucrose consumption ratio at day 43 after the CUS. However, HJDT $8 \mathrm{~g} / \mathrm{kg}$ had no effect on the sucrose consumption ratio, compared with the CUS depression group.

3.4. HJDT Improved the Locomotor Activity in OFT after the CUS. After the CUS, the total traveled distance in experimental groups, exposed to the CUS, decreased in OFT as compared with the normal control group (Figure 4(a)). The results in Figures 4(b)-4(d) showed that CUS depression rats decreased the rearing number, central time, and central distance ratio in OFT. As expected, paroxetine and HJDT $2 \mathrm{~g} / \mathrm{kg}$ treatment increased the rearing number, whereas there was no effect in rats treated with HJDT 4 and HJDT $8 \mathrm{~g} / \mathrm{kg}$ (Figure 4(b)). Also one-way ANOVA revealed that rats treated with paroxetine and HJDT ( 2 and $4 \mathrm{~g} / \mathrm{kg}$ ) increased the central time and central distance ratio in OFT while HJDT $8 \mathrm{~g} / \mathrm{kg}$ had no effects (Figures 4(c) and 4(d)).

3.5. HJDT Improved the Cognitive Decline in NORT after the CUS. As shown in Figure 5(a), there was no difference in the exploration time in $\mathrm{T}_{1}$ phase among groups after the 


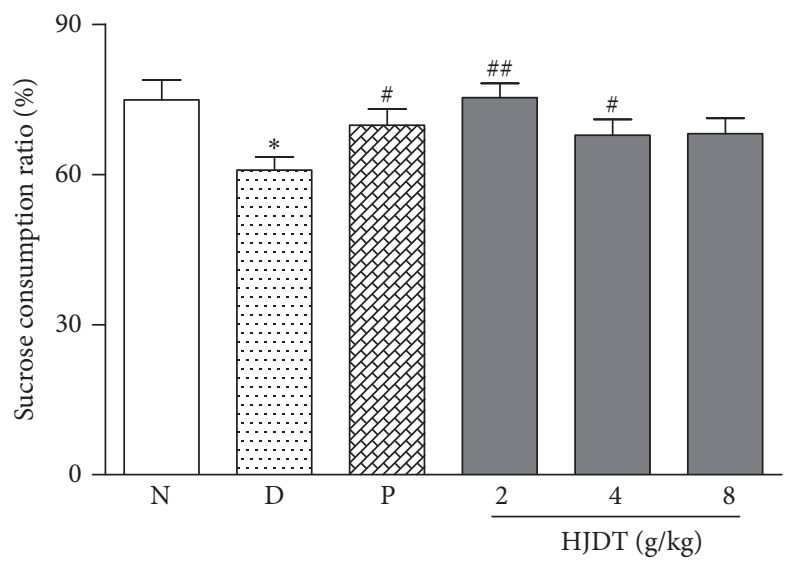

FIgURE 3: The effect of HJDT on the sucrose consumption ratio in SPT after the CUS. The sucrose consumption ratio decreased in the CUS depression group. Treatment with paroxetine and HJDT $(2,4 \mathrm{~g} / \mathrm{kg})$ increased the sucrose consumption ratio. ${ }^{*} p<0.05$ relative to the normal control group; ${ }^{\#} p<0.05$ and ${ }^{\# \#} p<0.01$ relative to the CUS depression group. N: normal control group; D: CUS depression group; P: paroxetine group; HJDT 2: HJDT $2 \mathrm{~g} / \mathrm{kg}$ group; HJDT 4: HJDT $4 \mathrm{~g} / \mathrm{kg}$ group; HJDT 8: HJDT $8 \mathrm{~g} / \mathrm{kg}$ group.

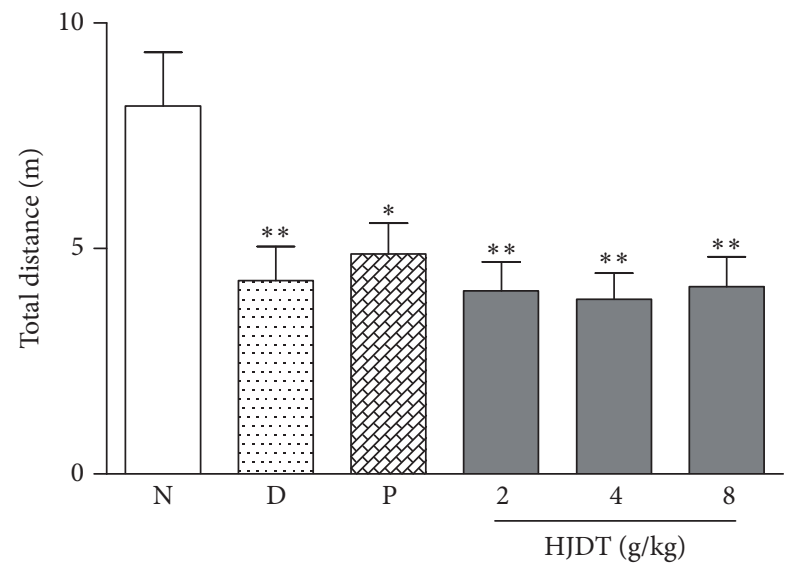

(a)

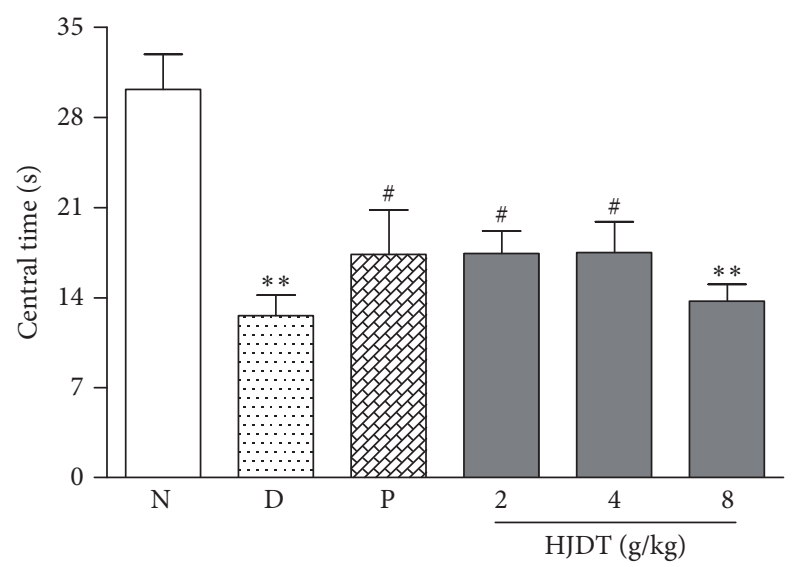

(c)

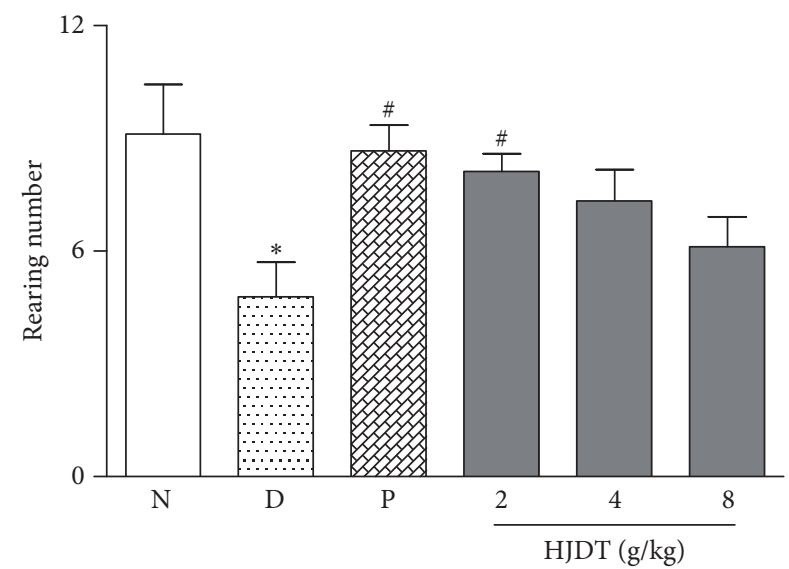

(b)

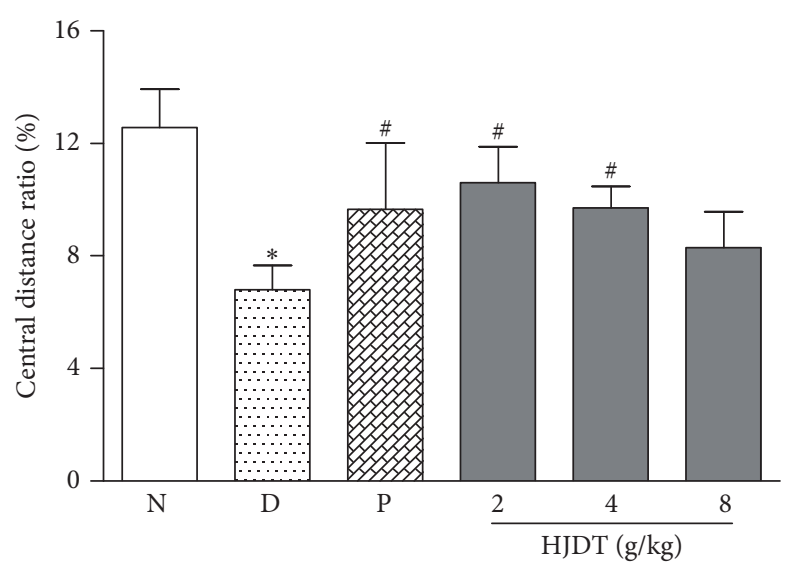

(d)

FIGURE 4: Effects of HJDT on the locomotor activity in OFT after the CUS. Traveled total distance decreased in all rats when exposed to CUS (a). The rearing number decreased in CUS depression group and was reversed with paroxetine and HJDT $2 \mathrm{~g} / \mathrm{kg}$ treatment (b). The central time and central distance ratio both decreased in CUS depression group and reversed with paroxetine and HJDT $(2,4 \mathrm{~g} / \mathrm{kg})$ treatment $(\mathrm{c}, \mathrm{d})$. ${ }^{*} p<0.05$ and ${ }^{* *} p<0.05$ relative to the normal control group; ${ }^{*} p<0.05$ relative to the CUS depression group. N: normal control group; D: CUS depression group; P: paroxetine group; HJDT 2: HJDT 2 g/kg group; HJDT 4: HJDT 4 g/kg group; HJDT 8: HJDT 8 g/kg group. 


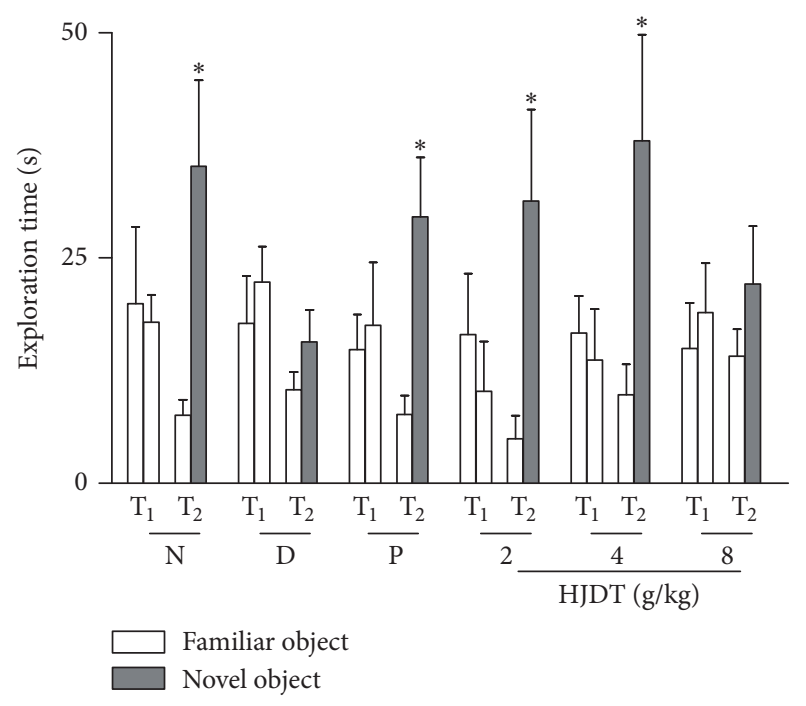

(a)
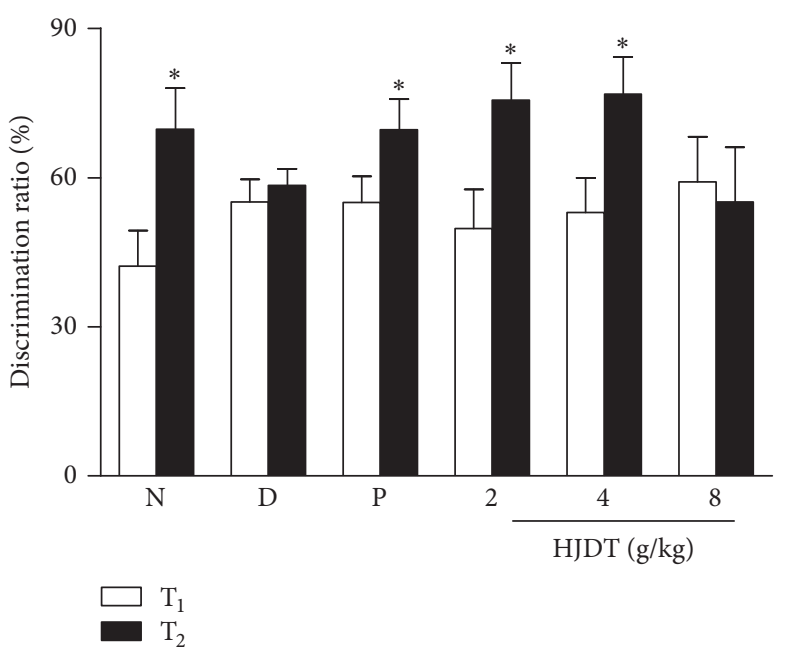

(b)

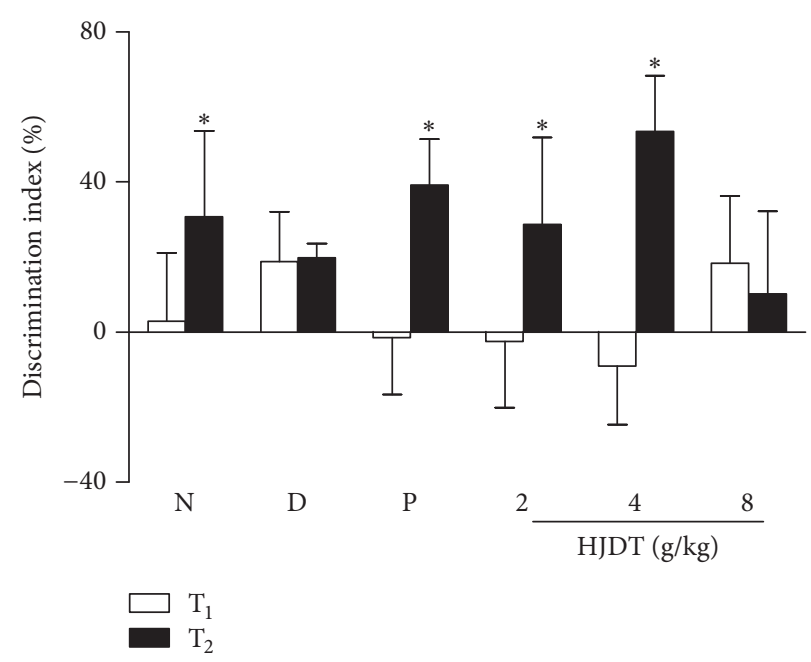

(c)

FIGURE 5: Effects of HJDT on the exploration time, DR, and DI in NORT after the CUS. The exploration time to novel object increased compared with familiar object in $\mathrm{T}_{2}$ phase in normal control group, paroxetine group, and HJDT (2, $\left.4 \mathrm{~g} / \mathrm{kg}\right)$ groups (a). DR (b) and DI (c) in $\mathrm{T}_{2}$ phase significantly increased in normal control group, paroxetine group, and HJDT $(2,4 \mathrm{~g} / \mathrm{kg})$ groups compared to $\mathrm{T}_{1}$ phase. ${ }^{*} p<0.05$ relative to the familiar object in $\mathrm{T}_{2}$ test $(\mathrm{a}) ;{ }^{*} \mathrm{p}<0.05$ relative to $\mathrm{T}_{1}$ phase in the same group (b-c). N: normal control group; $\mathrm{D}$ : CUS depression group; P: paroxetine group; HJDT 2: HJDT 2 g/kg group; HJDT 4: HJDT 4 g/kg group; HJDT 8: HJDT 8 g/kg group.

CUS. However, in $\mathrm{T}_{2}$ phase, the exploration time to the novel object was longer than to the familiar object in the normal control group, paroxetine group, and HJDT (2 and $4 \mathrm{~g} / \mathrm{kg}$ ) groups. CUS depression group and HJDT $8 \mathrm{~g} / \mathrm{kg}$ group had no difference in the exploration time between the familiar object and the novel object in $\mathrm{T}_{2}$ phase. Figures 5(b) and 5(c) showed there were no differences in the DR and the DI in $\mathrm{T}_{1}$ phase among all groups after the CUS. However, the DR and the $\mathrm{DI}$ in $\mathrm{T}_{2}$ phase in normal control group, paroxetine group, and HJDT (2 and $4 \mathrm{~g} / \mathrm{kg}$ ) groups showed significant increase (Figures 5(b) and 5(c)). CUS depression group and HJDT $8 \mathrm{~g} / \mathrm{kg}$ group had no differences in the DR and the DI in $\mathrm{T}_{2}$ phase when compared to $\mathrm{T}_{1}$ phase. It suggested that HJDT
( 2 and $4 \mathrm{~g} / \mathrm{kg}$ ) and paroxetine could improve the cognitive decline induced by CUS.

3.6. HJDT Decreased the Immobility Time in FST after the CUS. The immobility time increased in the CUS depression group compared with the normal control group (Figure 6). HJDT ( 2 and $4 \mathrm{~g} / \mathrm{kg}$ ) and paroxetine treatment reversed the increase of immobility time induced by the CUS.

3.7. HJDT Decreased the Microglia Number in the Hippocampal CA1 Region and DG. Iba-1 positive cell represents the activated microglia. The results of immunohistochemistry detection revealed the morphological and number change 


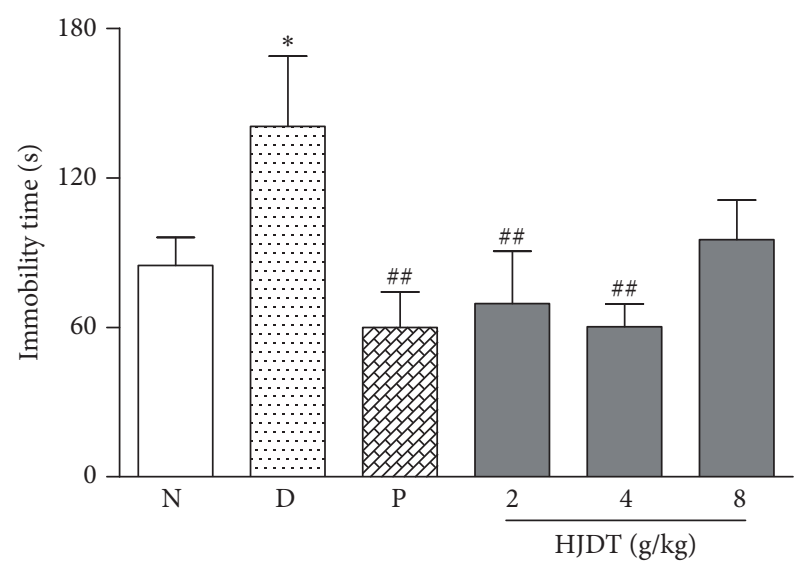

FIGURE 6: Effect of HJDT on the immobility time in FST after the CUS. The immobility time increased in CUS depression group and was reversed after treatment with paroxetine and HJDT $(2,4 \mathrm{~g} / \mathrm{kg})$. ${ }^{*} p<0.05$ relative to the normal control group; ${ }^{\# \#} p<0.01$ relative to the CUS depression group. N: normal control group; D: CUS depression group; P: paroxetine group; HJDT 2: HJDT $2 \mathrm{~g} / \mathrm{kg}$ group; HJDT 4: HJDT 4 g/kg group; HJDT 8: HJDT 8 g/kg group.

of microglia (Figure 7(a)). Figures $7(\mathrm{~b})$ and $7(\mathrm{c})$ showed the microglia number increased in both hippocampal CA1 region and DG after the CUS. Treatment with paroxetine and HJDT $2 \mathrm{~g} / \mathrm{kg}$ decreased the microglia number in hippocampal CA1 region and DG (Figures 7(b) and 7(c)). HJDT $4 \mathrm{~g} / \mathrm{kg}$ decreased microglia number only in hippocampal CA1 region, but not in DG (Figure 7(b)). However, HJDT $8 \mathrm{~g} / \mathrm{kg}$ had no effect on the microglia number in both the hippocampal CA1 region and DG (Figures 7(b) and 7(c)).

3.8. HJDT Increased the BDNF, TrkB, and $p C R E B / C R E B$ Expression in the Hippocampus after the CUS. BDNF, TrkB, CREB, and pCREB protein expression was detected by Western-blotting method (Figure 8(d)). Results showed the BDNF, TrkB, and $\mathrm{pCREB} / \mathrm{CREB}$ expression decreased in the CUS depression group, compared with the normal control group (Figures $8(\mathrm{a})-8(\mathrm{c})$ ). After treatment with paroxetine or HJDT ( 2 and $4 \mathrm{~g} / \mathrm{kg}$ ), BDNF, TrkB, and pCREB/CREB expression increased (Figures 8(a)-8(c)).

3.9. HJDT Enhanced the PCREB DNA Activity in the Hippocampus. The pCREB $\mathrm{OD}_{593}$ value in the normal control group was $2.77 \pm 0.09$. But in the CUS depression group, it decreased to $2.08 \pm 0.03$. After treatment with HJDT ( 2 and $4 \mathrm{~g} / \mathrm{kg}$ ), it increased to $2.66 \pm 0.10$ and $2.63 \pm 0.09$, respectively (Figure 9). It suggested the pCREB DNA activity improved. Compared with the CUS depression group, paroxetine and HJDT $8 \mathrm{~g} / \mathrm{kg}$ did not affect the pCREB DNA activity (Figure 9).

3.10. HJDT Decreased the Concentration of TNF- $\alpha$, iNOS, and IL-6/IL-10 Ratio in the Hippocampus. The results of RT-PCR showed that CUS depression model was related with inflammation reaction. The mRNA of proinflammatory cytokines, IL-1 $\beta$, TNF- $\alpha$, and IL-6/IL-10 ratio greatly increased in the hippocampus after the CUS. Paroxetine only reversed the increase of iNOS and TNF- $\alpha$ in the hippocampus (Figures $10(\mathrm{a})$ and $10(\mathrm{~d})$ ), whereas there was no effect on IL-6/IL10 ratio and IL-1 $\beta$ (Figures $10(\mathrm{~b})$ and $10(\mathrm{c})$ ). HJDT (2 and $4 \mathrm{~g} / \mathrm{kg}$ ) treatment reversed the increase of iNOS, IL-6/IL10 , and TNF- $\alpha$ (Figures 10(a), 10(b), and 10(d)). Also HJDT $8 \mathrm{~g} / \mathrm{kg}$ reduced the iNOS mRNA (Figure 10(a)), but there was no effect on the other mRNA of inflammatory cytokines.

3.11. HJDT Had No Effect on the 5-HT and DA Contents in the Hippocampus. The results of ELISA showed the contents of 5-HT and DA both decreased in the CUS depression group when compared with the normal control group. Treatment with paroxetine reversed the decrease of 5-HT content (Figure 11(a)), but not DA content (Figure 11(b)) in the hippocampus. But HJDT (2, 4, and $8 \mathrm{~g} / \mathrm{kg})$ groups had no effects on the contents of 5-HT and DA in the hippocampus.

\section{Discussion}

The present study was aimed at examining the depressionlike response following administration of aqueous extract of HJDT in rats which were subjected to the CUS. The major findings of this study include the fact that administration of HJDT during the CUS ameliorated depressionlike behaviors, like anhedonia, decreased locomotor activity, despair condition, and cognitive function deficit, which could be relative to the inhibition of microglia activation, following with inflammation reaction inhibition and BDNFTrkB-CREB pathway upregulation.

The stress-induced body weight decrease is an accompanying symptom of depression. However, HJDT did not gain the body weight decrease in CUS-treated rats except paroxetine treatment from day 35 to day 42 after the CUS, which is consistent with the previous findings about Traditional Chinese Medicine (TCM) treatment $[19,20]$. The phenomenon of this change may be related to miss of the best period of growth and development in rats, which is an irreversible inhibition caused by the CUS. FST has been commonly used to evaluate the despair condition after stress and screen for potential antidepressants. Combined with no effects on body weight after HJDT treatment in our results, it suggested that the decreased immobility time in FST was unrelated with body weight. A reduction of sucrose consumption reflects anhedonia, which is one of the core symptoms of depression [21]. And this change could be restored by antidepressant treatment $[22,23]$. The same results were found after HJDT treatment in FST. The decrease of total traveled distance, central distance ratio, and central time in OFT indicate a lower desire to explore, which may mimic psychomotor retardation [24]. Additionally, the low levels of rearing in OFT, and the increased immobility time in FST are often used as indices of a despair state $[25,26]$. The rats exhibited improvement of depressive-like behaviors in OFT and in FST after HJDT or paroxetine treatments. The stress-induced cognitive decline is also an accompanying symptom of depression, which can be evaluated by Morris water maze task, the radial arm maze task [27, 28], 
CA1
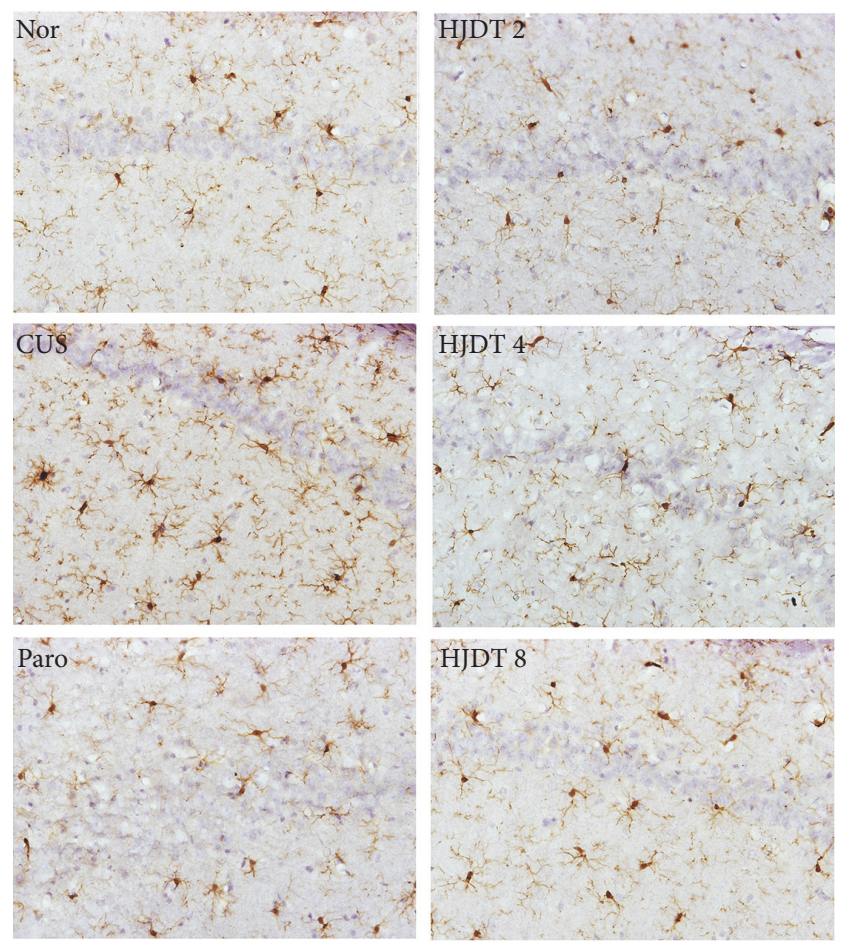

(a)

(a)

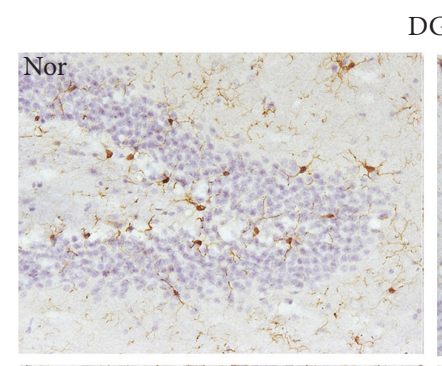

DG
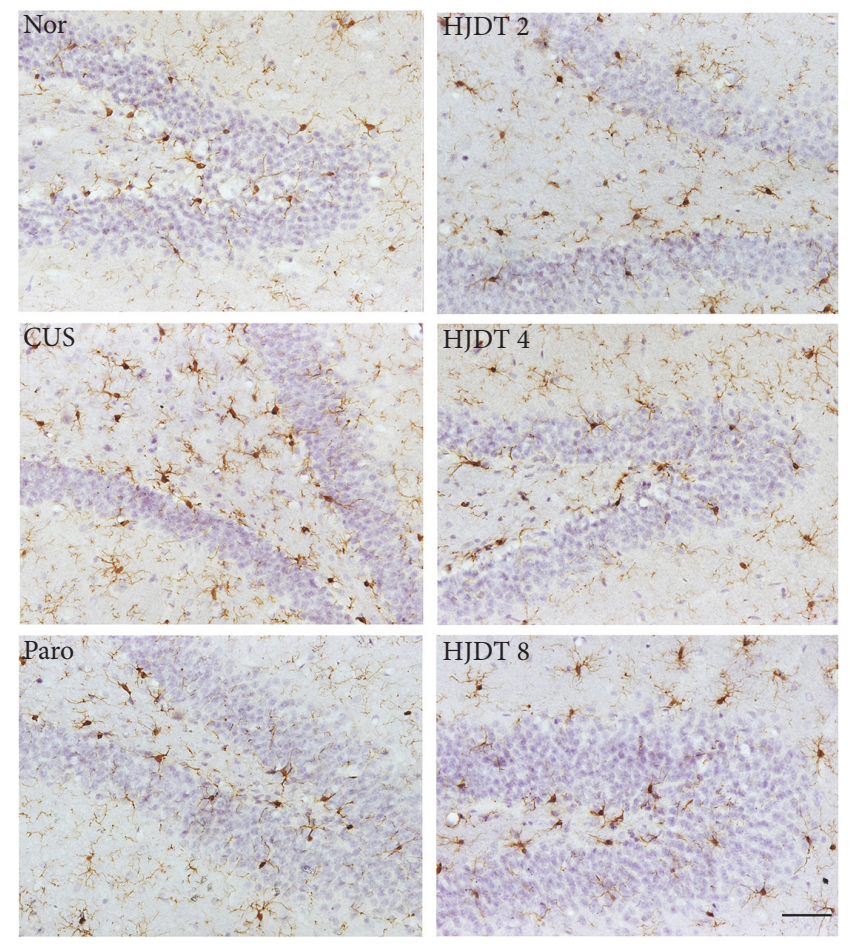

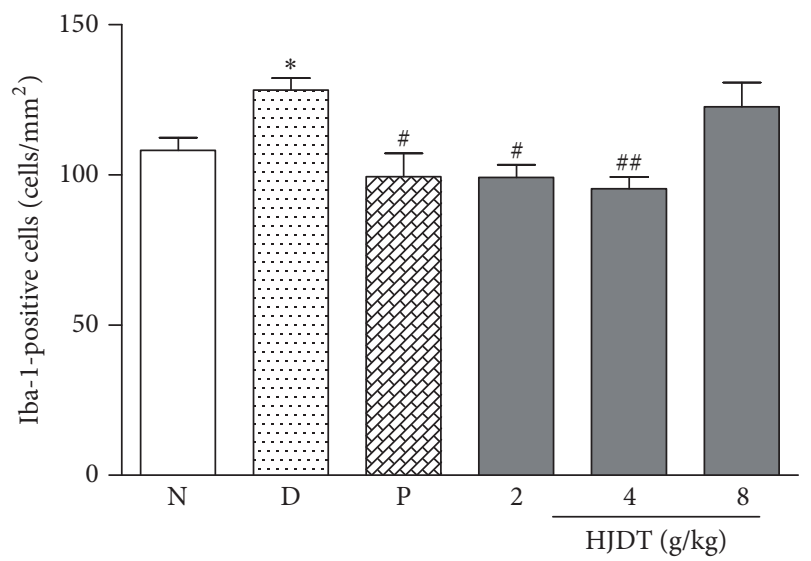

(b)

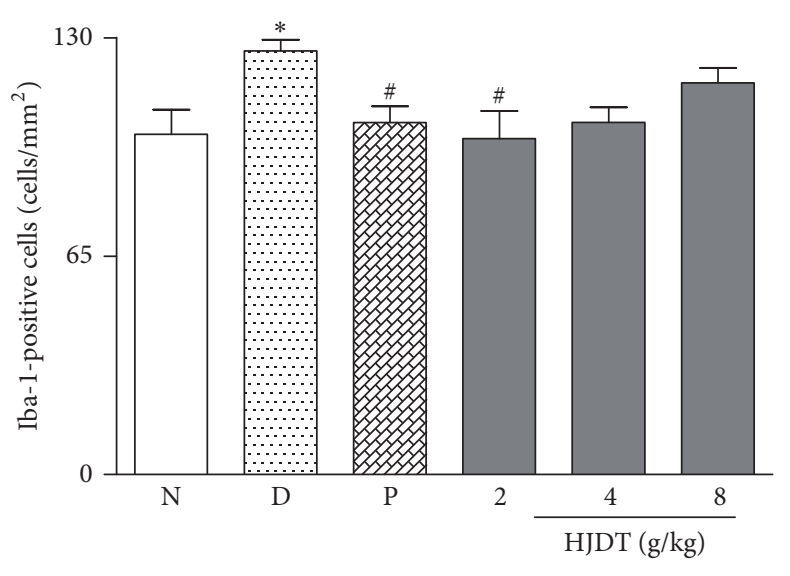

(c)

FIGURE 7: Effect of HJDT on the microglia morphology and number in the hippocampal CA1 region and DG after the CUS. The representative morphology and the number change of microglia in all groups (a). The number of Iba-1 positive cells in hippocampal CA1 region increased in CUS depression group and was reversed with paroxetine and HJDT $(2,4 \mathrm{~g} / \mathrm{kg})$ treatments (b). The number of Iba-1 positive cells in hippocampus DG increased in CUS depression group and was reversed with paroxetine and HJDT $2 \mathrm{~g} / \mathrm{kg}$ treatment $(\mathrm{c}) .{ }^{*} p<0.05 \mathrm{relative}$ to the normal control group; ${ }^{\#} p<0.05$ and ${ }^{\# \#} p<0.01$ relative to the CUS depression group. Nor/N: normal control group; CUS/D: CUS depression group; Paro/P: paroxetine group; HJDT 2: HJDT $2 \mathrm{~g} / \mathrm{kg}$ group; HJDT 4: HJDT $4 \mathrm{~g} / \mathrm{kg}$ group; HJDT 8: HJDT 8 g/kg group. Scale bar $=100 \mu \mathrm{m}, \times 200$.

and others. The NORT, less dependent on locomotion and reflecting both frontal cortical and hippocampal function, as well as a fear-motivated learning task, is often used in the cognitive impairment diseases $[29,30]$. Our results showed the significant cognitive impairment in the CUS depression rats. HJDT ( 2 and $4 \mathrm{~g} / \mathrm{kg}$ ) and paroxetine increased the DR, DI, and the exploration time to novel object, which indicated the improvement of the cognitive performance.
The classic opinion on the development of depression considered that it was related to the monoamine neurotransmitter and serotonin (5-HT) system dysfunction [31, 32]. So at present the commonly used antidepressant drugs in clinic include selective 5-HT reuptake inhibitors, NA reuptake inhibitors, 5-HT and NA dual reuptake inhibitors. However, there was no effect of HJDT on the DA and 5-HT contents in our study, which suggested the improvement of HJDT on the 


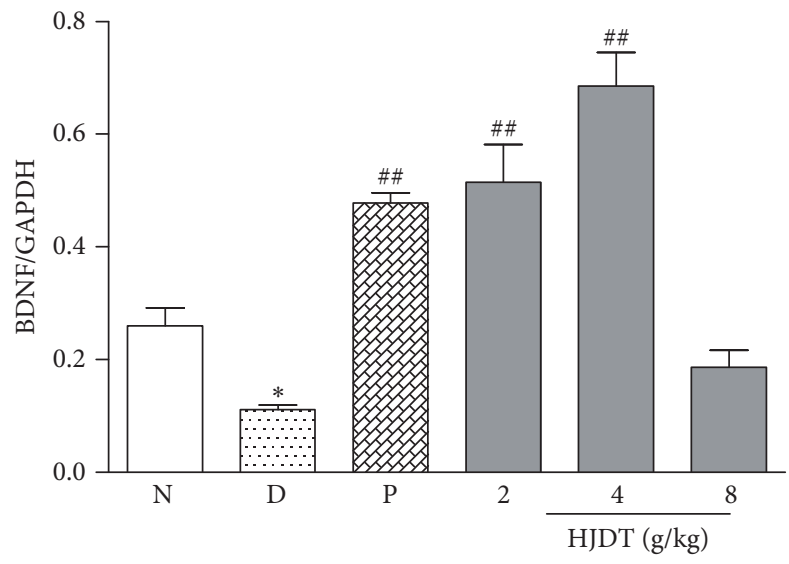

(a)

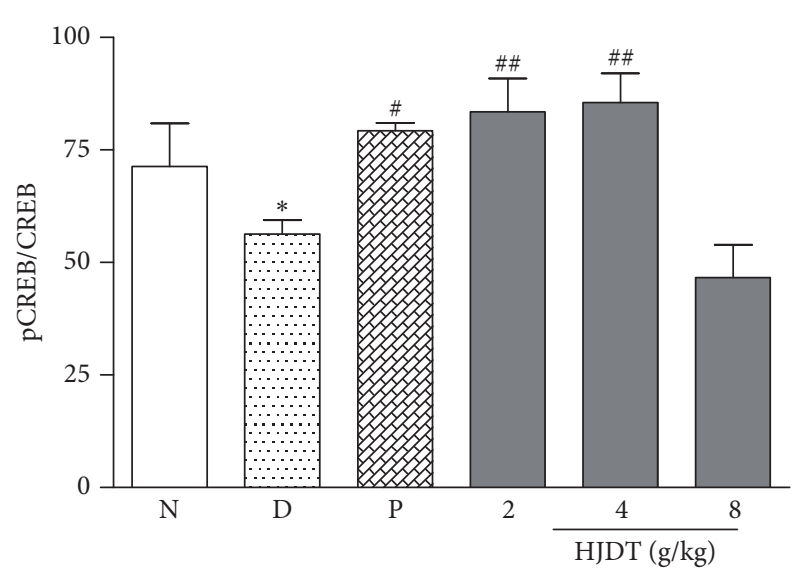

(c)

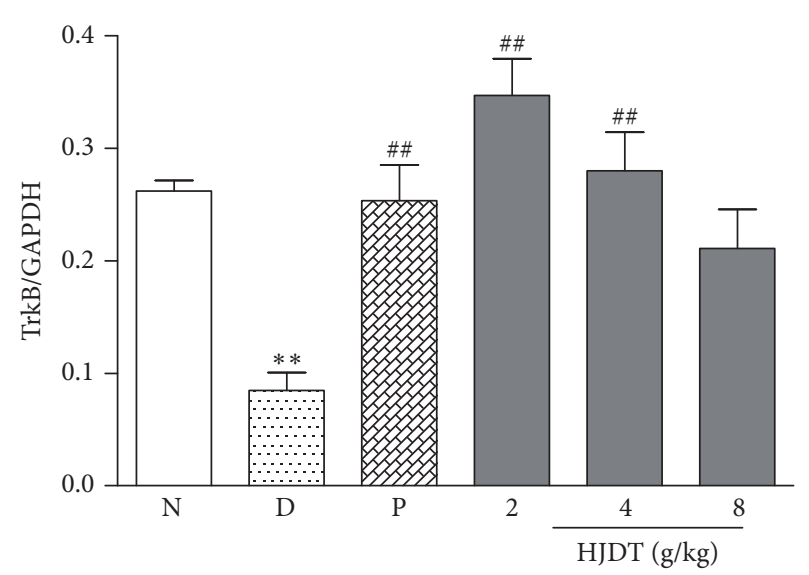

(b)

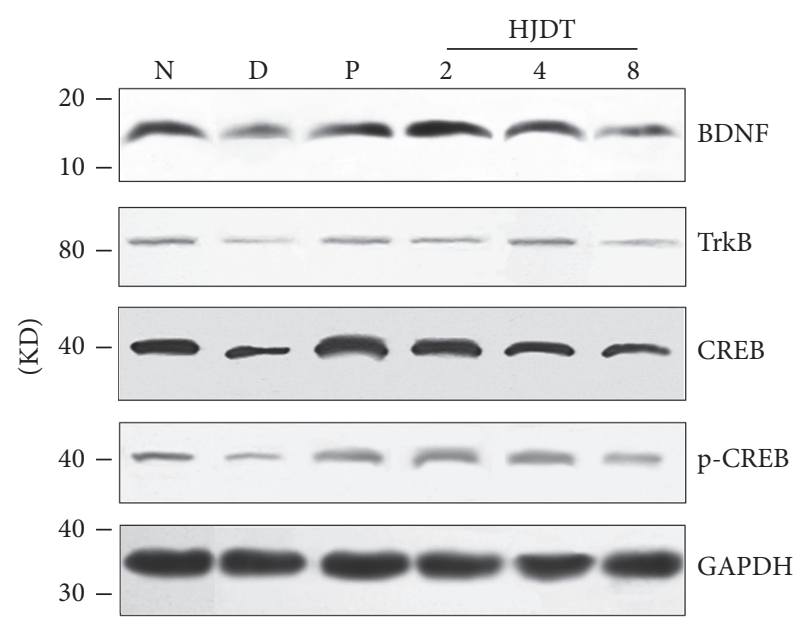

(d)

FigurE 8: Effect of HJDT on the BDNF, TrkB, and pCREB/CREB expression in the hippocampus after the CUS. The expression of BDNF (a), TrkB (b), and pCREB/CREB (c) decreased in the CUS depression group and was reversed with paroxetine and HJDT (2, $4 \mathrm{~g} / \mathrm{kg})$ treatment. Protein expression is induced by CUS and drug treatment in the hippocampus (d). ${ }^{*} p<0.05$ and ${ }^{* *} p<0.01$ relative to the normal control group; ${ }^{\#} p<0.05$ and ${ }^{\# \#} p<0.01$ relative to the CUS depression group. N: normal control group; D: CUS depression group; P: paroxetine group; HJDT 2: HJDT $2 \mathrm{~g} / \mathrm{kg}$ group; HJDT 4: HJDT $4 \mathrm{~g} / \mathrm{kg}$ group; HJDT 8: HJDT $8 \mathrm{~g} / \mathrm{kg}$ group.

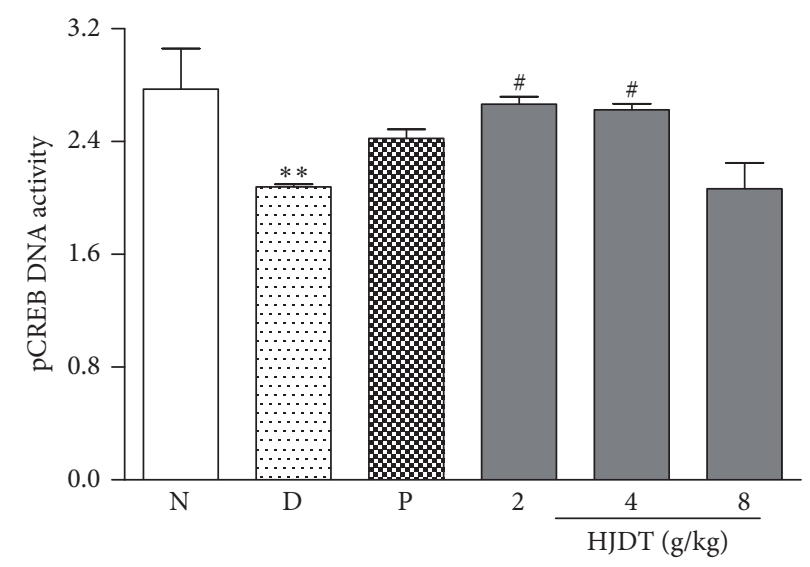

FIGURE 9: Effect of HJDT on the pCREB DNA activity in the hippocampus after the CUS. The pCREB DNA activity decreased in the hippocampus and was reversed with HJDT $(2,4 \mathrm{~g} / \mathrm{kg})$ treatment and there was no effect with paroxetine treatment. ${ }^{* *} p<0.01$ relative to the normal control group; ${ }^{\#} p<0.05$ relative to the CUS depression group. N: normal control group; D: CUS depression group; P: paroxetine group; HJDT 2: HJDT 2 g/kg group; HJDT 4: HJDT 4 g/kg group; HJDT 8: HJDT 8 g/kg group. 


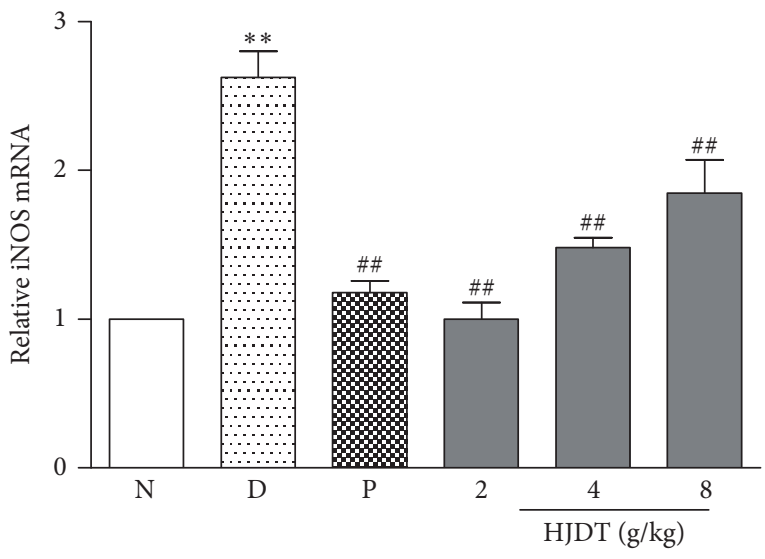

(a)

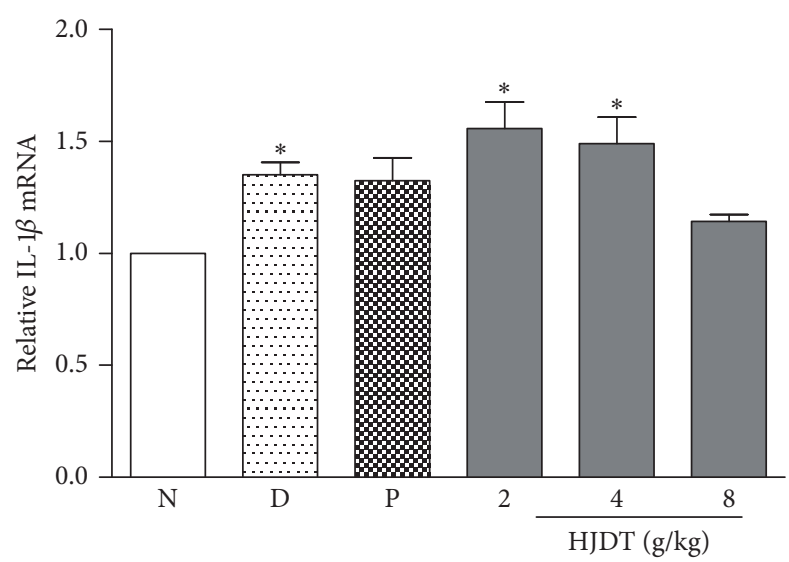

(c)

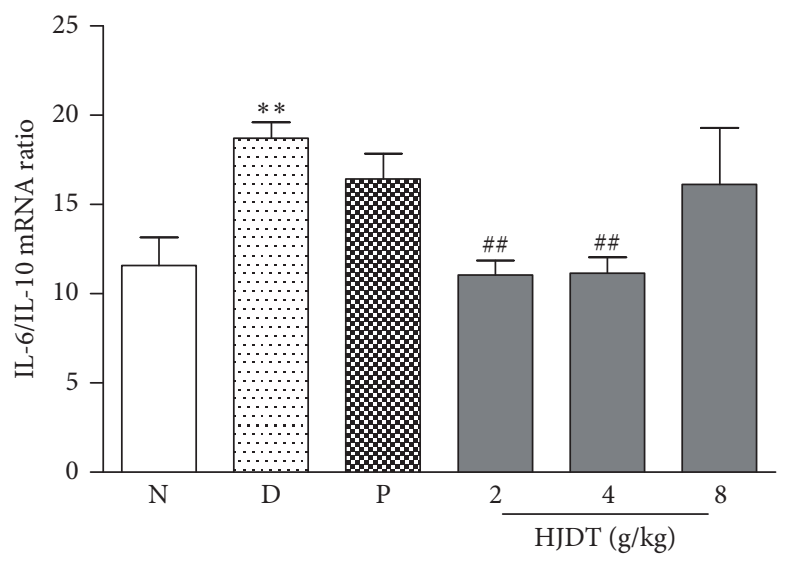

(b)

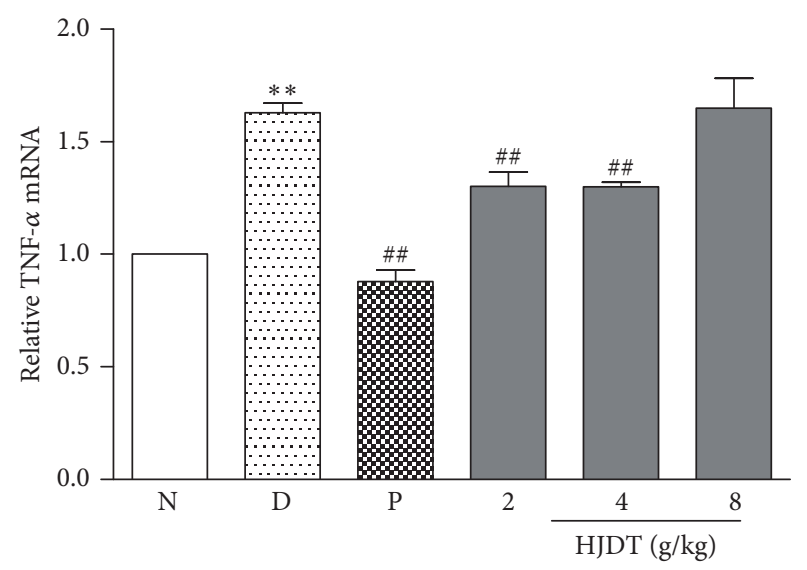

(d)

FIGURE 10: Effect of HJDT on proinflammatory and anti-inflammatory cytokines mRNA in the hippocampus after the CUS. The effects of HJDT on the iNOS mRNA (a), IL-6/IL-10 mRNA ratio (b), IL-1 $\beta$ mRNA (c), and TNF- $\alpha$ mRNA (d) in the hippocampus. ${ }^{*} p<0.05$ and ${ }^{* *} p<0.01$ relative to the normal control group; ${ }^{\# \#} p<0.01$ relative to the CUS depression group. N: normal control group; D: CUS depression group; P: paroxetine group; HJDT 2: HJDT 2 g/kg group; HJDT 4: HJDT $4 \mathrm{~g} / \mathrm{kg}$ group; HJDT 8: HJDT $8 \mathrm{~g} / \mathrm{kg}$ group.

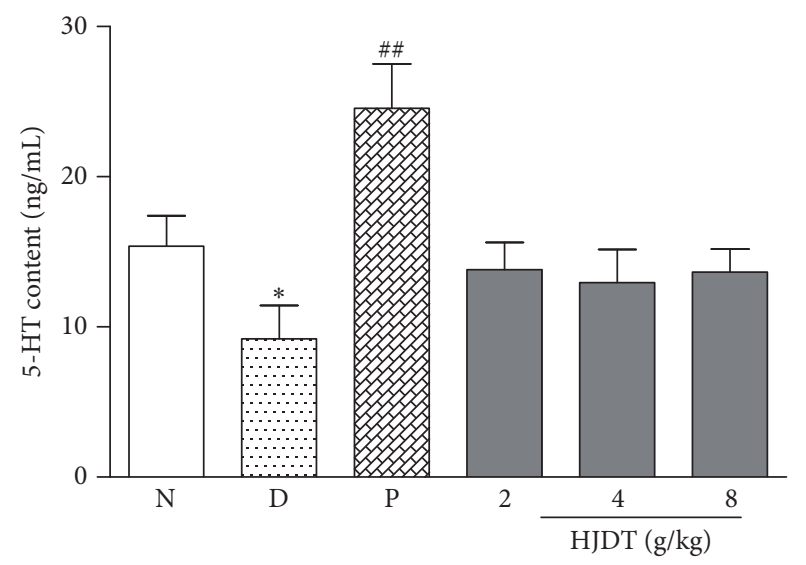

(a)

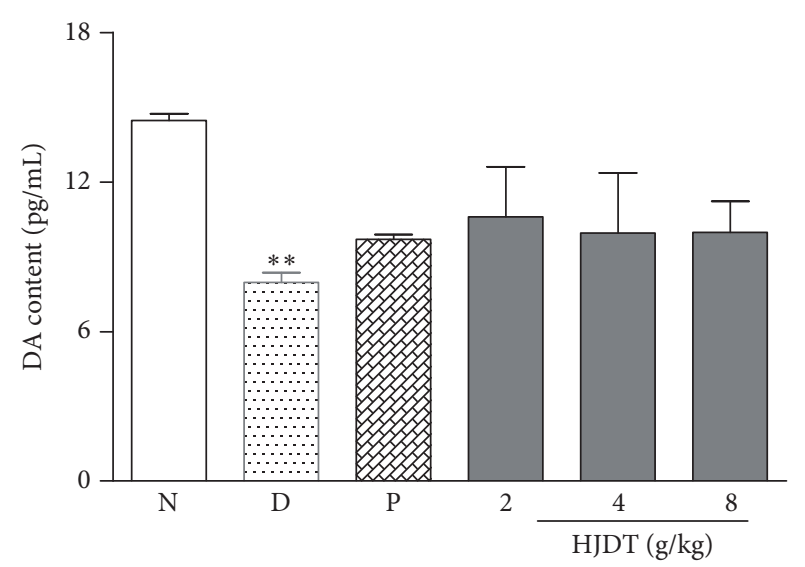

(b)

FIGURE 11: Effect of HJDT on the 5-HT and DA contents in the hippocampus after the CUS. The contents of 5-HT (a) and DA (b) decreased in CUS depression group. Paroxetine treatment only increased the 5-HT content (a) and there was no effect on DA content (b) in the hippocampus. There was no effect of HJDT on the contents of 5-HT and DA in the hippocampus (a, b). ${ }^{*} p<0.05$ and ${ }^{* *} p<0.01$ relative to the normal control group; ${ }^{\# \#} p<0.01$ relative to the CUS depression group. N: normal control group; D: CUS depression group; P: paroxetine group; HJDT 2: HJDT 2 g/kg group; HJDT 4: HJDT 4 g/kg group; HJDT 8: HJDT 8 g/kg group. 
depression-like behaviors may not involve the monoamine neurotransmitters.

Neuroinflammation is considered as one of the common pathogeneses of depression in recent research. The activation of the inflammatory immune system may induce the decrease of neurons regeneration and neurotrophic factors release and enhancement of the neuroinflammation reaction [33, 34]. Microglia are the major innate immune cell population in brain tissue and microglia-mediated inflammation is associated with the pathogenesis of various neuronal disorders, like depression, which are major cellular sources of TNF and IL-1 family of cytokines $[35,36]$. Furthermore, several researches have reported that microglia cells in the brain were obviously activated in the brain of suicide victims [37], and antidepressants fluoxetine, paroxetine, and sertraline inhibited the microglial activation and reduced the release of inflammatory cytokines $[38,39]$. Our study found that HJDT ( 2 and $4 \mathrm{~g} / \mathrm{kg}$ ) decreased the microglia number in the hippocampus, following a reduced production of proinflammatory cytokines, including iNOS, TNF- $\alpha$, and IL-6/IL-10 ratio instead of IL-1 $\beta$. iNOS is inflammation inducible, which has been considered as an important role in the depressive behavior induced by chronic stress exposure [40]. Recently, Peng et al. reported the iNOS inhibitor $1400 \mathrm{~W}$ exhibited a significant protection on neurons compared with CUS group [41]. TNF- $\alpha$, IL-6, and IL-10 also played important roles in depression induced by stress, which caused the increase of proinflammation cytokines, including TNF- $\alpha$, IL- 6 , and IL$1 \beta$ in serum and hippocampus $[24,42]$. Our results have confirmed that HJDT decreased the microglia number and the proinflammation cytokines concentration. But HJDT had no effect on the elevation of IL-1 $\beta$ after CUS, which was contrary with some reports $[43,44]$. It may be related with the sensitivity of HJDT to different proinflammation cytokines.

In addition, activation of microglia can provoke the dysregulation of several growth factors in depression, like BDNF. BDNF and CREB had been reported to involve neuronal differentiation and survival as well as the synaptic plasticity associated with learning and memory in various nervous system disorders, including depression $[45,46]$. It has been reported that chronic stress caused a downregulation of hippocampal BDNF or CREB levels and this reduction could be upregulated through antidepressant therapy [47, 48]. Our study found the expression of BDNF and its receptor TrkB as well as pCREB/CREB ratio decreased. It is worth mentioning that the pCREB DNA activity also decreased after CUS. Upregulation of the expression of BDNF-TrkB-CREB and the pCREB DNA activity in the hippocampus could contribute to the antidepressant effects of HJDT, manifested as improvement of depression-like behaviors.

\section{Conclusion}

In summary, the present study supports the notion that microglial activation, followed by inflammation reaction and BDNF decreasing, may contribute to the depressive-like behavior in a rat model in response to the CUS. HJDT treatment potentially ameliorated the depression-like behaviors. Additionally the research indicated that the effects of
HJDT might be related to the decrease of microglia number, following with the reduction of inflammatory cytokines and the promotion of BDNF-TrkB-CREB pathway, which was independent of the HT and DA contents change. This antidepressant effect of HJDT was probably related to the synergistic effect of each active component, including berberine, baicalin, wogonoside, and gardenoside $[8,9]$.

\section{Conflicts of Interest}

No conflict of financial interests exists.

\section{Acknowledgments}

This work was supported by grants from the Scientific Foundation in TCM of Zhejiang Province (2013ZB028) and the Foundation of Science Technology Department of Zhejiang Province (2016C37099). The authors acknowledge GlaxoSmithKline, England, for providing paroxetine.

\section{References}

[1] G. Masi and P. Brovedani, "The hippocampus, neurotrophic factors and depression: possible implications for the pharmacotherapy of depression," CNS Drugs, vol. 25, no. 11, pp. 913-931, 2011.

[2] S.-W. Park, Y.-K. Kim, J.-G. Lee et al., "Antidepressant-like effects of the traditional Chinese medicine kami-shoyo-san in rats," Psychiatry and Clinical Neurosciences, vol. 61, no. 4, pp. 401-406, 2007.

[3] Y. Ohta, T. Kobayashi, K. Nishida, E. Sasaki, and I. Ishiguro, "Preventive effect of Oren-gedoku-to (Huanglian-Jie-Du-Tang) extract on the development of stress-induced acute gastric mucosal lesions in rats," Journal of Ethnopharmacology, vol. 67, no. 3, pp. 377-384, 1999.

[4] Y. Ohta, M. Kongo-Nishimura, T. Hayashi, and T. Kishikawa, "Effect of Oren-gedoku-to (Huanglian-Jie-Du-Tang) extract on disruption of hepatic antioxidant defense systems in rats treated with D-galactosamine," Journal of Ethnopharmacology, vol. 94, no. 2-3, pp. 323-329, 2004.

[5] S. S. K. Durairajan, Y.-Y. Huang, P.-Y. Yuen et al., "Effects of huanglian-jie-du-tang and its modified formula on the modulation of amyloid- $\beta$ precursor protein processing in alzheimer's disease models," PLoS ONE, vol. 9, no. 3, Article ID e92954, 2014.

[6] M.-F. Zeng, L.-M. Pan, H.-X. Zhu, Q.-C. Zhang, and L.-W. Guo, "Comparative pharmacokinetics of baicalin in plasma after oral administration of Huang-Lian-Jie-Du-Tang or pure baicalin in MCAO and sham-operated rats," Fitoterapia, vol. 81, no. 6, pp. 490-496, 2010.

[7] Y. Ye, C. Huang, L. Jiang et al., "Huanglian-Jie-Du-Tang extract protects against chronic brain injury after focal cerebral ischemia via hypoxia-inducible-factor- $1 \alpha$-regulated vascular endothelial growth factor signaling in mice," Biological and Pharmaceutical Bulletin, vol. 35, no. 3, pp. 355-361, 2012.

[8] B. Lee, B. Sur, M. Yeom, I. Shim, H. Lee, and D.-H. Hahm, "Effect of berberine on depression- and anxiety-like behaviors and activation of the noradrenergic system induced by development of morphine dependence in rats," Korean Journal of Physiology and Pharmacology, vol. 16, no. 6, pp. 379-386, 2012. 
[9] K. Zhang, X. Pan, F. Wang et al., "Baicalin promotes hippocampal neurogenesis via SGK1- and FKBP5-mediated glucocorticoid receptor phosphorylation in a neuroendocrine mouse model of anxiety/depression," Scientific Reports, vol. 6, Article ID 30951, 2016.

[10] V. H. Perry and S. Gordon, "Macrophages and microglia in the nervous system," Trends in Neurosciences, vol. 11, no. 6, pp. 273277, 1988.

[11] H. Kettenmann, U. K. Hanisch, M. Noda, and A. Verkhratsky, "Physiology of microglia," Physiological Reviews, vol. 91, no. 2, pp. 461-553, 2011.

[12] R. Yirmiya, N. Rimmerman, and R. Reshef, "Depression as a microglial disease," Trends in Neurosciences, vol. 38, no. 10, pp. 637-658, 2015.

[13] G. Milior, C. Lecours, L. Samson et al., "Fractalkine receptor deficiency impairs microglial and neuronal responsiveness to chronic stress," Brain, Behavior, and Immunity, 2015.

[14] C. Gomes, R. Ferreira, J. George et al., "Activation of microglial cells triggers a release of brain-derived neurotrophic factor (BDNF) inducing their proliferation in an adenosine $A_{2 A}$ receptor-dependent manner: $A_{2 A}$ receptor blockade prevents BDNF release and proliferation of microglia," Journal of Neuroinflammation, vol. 10, article 16, 2013.

[15] T. Yan, B. Wu, Z.-Z. Liao et al., "Brain-derived neurotrophic factor signaling mediates the antidepressant-like effect of the total flavonoids of alpiniae oxyphyllae fructus in chronic unpredictable mild stress mice," Phytotherapy Research, pp. 1493-1502, 2016.

[16] J. Grønli, C. Bramham, R. Murison et al., "Chronic mild stress inhibits BDNF protein expression and CREB activation in the dentate gyrus but not in the hippocampus proper," Pharmacology Biochemistry and Behavior, vol. 85, no. 4, pp. 842-849, 2006.

[17] H.-M. Qiu, J.-X. Yang, X.-H. Wu et al., "Antidepressive effect of paroxetine in a rat model: Upregulating expression of serotonin and norepinephrine transporter," NeuroReport, vol. 24, no. 10, pp. 520-525, 2013.

[18] P. Xu, K. Z. Wang, C. Lu et al., "Antidepressant-like effects and cognitive enhancement of the total phenols extract of Hemerocallis citrina Baroni in chronic unpredictable mild stress rats and its related mechanism," Journal of Ethnopharmacology, vol. 194, pp. 819-826, 2016.

[19] K. L. Weihs, E. C. Settle Jr., S. R. Batey, T. L. Houser, R. M. J. Donahue, and J. A. Ascher, "Bupropion sustained release versus paroxetine for the treatment of depression in the elderly," Journal of Clinical Psychiatry, vol. 61, no. 3, pp. 196-202, 2000.

[20] J. Lu, R.-H. Shao, L. Hu, Y. Tu, and J.-Y. Guo, "Potential antiinflammatory effects of acupuncture in a chronic stress model of depression in rats," Neuroscience Letters, vol. 618, pp. 31-38, 2016.

[21] J. L. Moreau, "Validation of an animal model of anhedonia, a core symptom of depression," Encephale, vol. 23, no. 4, pp. 280289, 1997.

[22] P. Yang, L. Li, X.-J. Liu et al., "Effect of Chaihu-Shugan-San on the mRNA expression of the 5-HT1A receptor and cellular proliferation in the hippocampus of epileptic rats with depression," Experimental and Therapeutic Medicine, vol. 11, no. 1, pp. 124-130, 2016.

[23] D. Zurawek, M. Kusmider, A. Faron-Gorecka et al., "Timedependent miR-16 serum fluctuations together with reciprocal changes in the expression level of miR-16 in mesocortical circuit contribute to stress resilient phenotype in chronic mild stress An animal model of depression," European Neuropsychopharmacology, vol. 26, no. 1, pp. 23-36, 2016.

[24] W. Tao, Y. Dong, Q. Su et al., "Liquiritigenin reverses depression-like behavior in unpredictable chronic mild stressinduced mice by regulating PI3K/Akt/mTOR mediated BDNF/ TrkB pathway," Behavioural Brain Research, vol. 308, pp. 177186, 2016.

[25] T. Yan, M. Xu, B. Wu et al., "The effect of Schisandra chinensis extracts on depression by noradrenergic, dopaminergic, GABAergic and glutamatergic systems in the forced swim test in mice," Food and Function, vol. 7, no. 6, pp. 2811-2819, 2016.

[26] C.-L. Su, C.-W. Su, Y.-H. Hsiao, and P.-W. Gean, "Epigenetic regulation of BDNF in the learned helplessness-induced animal model of depression," Journal of Psychiatric Research, vol. 76, pp. 101-110, 2016.

[27] S. S. Patel, V. Mehta, H. Changotra, and M. Udayabanu, "Depression mediates impaired glucose tolerance and cognitive dysfunction: A neuromodulatory role of rosiglitazone," Hormones and Behavior, vol. 78, pp. 200-210, 2016.

[28] F. Khojasteh, A. Nahavandi, S. Mehrpouya et al., "Cognitive impairment induced by permanent bilateral common carotid occlusion exacerbates depression-related behavioral, biochemical, immunological and neuronal markers," Brain Research, vol. 1596, pp. 58-68, 2015.

[29] M. D. Scofield, H. Trantham-Davidson, M. Schwendt et al., "Failure to recognize novelty after extended methamphetamine self-administration results from loss of long-term depression in the perirhinal cortex," Neuropsychopharmacology, vol. 40, no. 11, pp. 2526-2535, 2015.

[30] J. Wu, Z. Zhao, B. Sabirzhanov et al., "Spinal cord injury causes brain inflammation associated with cognitive and affective changes: role of cell cycle pathways," Journal of Neuroscience, vol. 34, no. 33, pp. 10989-11006, 2014.

[31] F. Darcet, A. M. Gardier, D. J. David, and J.-P. Guilloux, "Chronic 5-HT4 receptor agonist treatment restores learning and memory deficits in a neuroendocrine mouse model of anxiety/depression," Neuroscience Letters, vol. 616, pp. 197-203, 2016.

[32] P. Blier, "Neurobiology of depression and mechanism of action of depression treatments," The Journal of clinical psychiatry, vol. 77, no. 3, p. e319, 2016.

[33] S. Hashioka, P. L. McGeer, A. Monji, and S. Kanba, "Antiinflammatory effects of antidepressants: possibilities for preventives against alzheimer's disease," Central Nervous System Agents in Medicinal Chemistry, vol. 9, no. 1, pp. 12-19, 2009.

[34] S. Pålhagen, H. Qi, B. Mårtensson, J. Wålinder, A.-K. Granérus, and P. Svenningsson, "Monoamines, BDNF, IL-6 and corticosterone in CSF in patients with Parkinson's disease and major depression," Journal of Neurology, vol. 257, no. 4, pp. 524-532, 2010.

[35] M. Boehme, M. Guenther, A. Stahr et al., "Impact of indomethacin on neuroinflammation and hippocampal neurogenesis in aged mice," Neuroscience Letters, vol. 572, pp. 7-12, 2014.

[36] S. Hellwig, S. Brioschi, S. Dieni et al., "Altered microglia morphology and higher resilience to stress-induced depressionlike behavior in CX3CR1-deficient mice," Brain, Behavior, and Immunity, vol. 55, pp. 126-137, 2016.

[37] J. Steiner, H. Bielau, R. Brisch et al., "Immunological aspects in the neurobiology of suicide: elevated microglial density 
in schizophrenia and depression is associated with suicide," Journal of Psychiatric Research, vol. 42, no. 2, pp. 151-157, 2008.

[38] C.-M. Lim, S.-W. Kim, J.-Y. Park, C. Kim, S. H. Yoon, and J.-K. Lee, "Fluoxetine affords robust neuroprotection in the postischemic brain via its anti-inflammatory effect," Journal of Neuroscience Research, vol. 87, no. 4, pp. 1037-1045, 2009.

[39] H. Horikawa, T. A. Kato, Y. Mizoguchi et al., "Inhibitory effects of SSRIs on IFN- $\gamma$ induced microglial activation through the regulation of intracellular calcium," Progress in NeuroPsychopharmacology and Biological Psychiatry, vol. 34, no. 7, pp. 1306-1316, 2010.

[40] D. Wang, S. C. An, and X. Zhang, "Prevention of chronic stressinduced depression-like behavior by inducible nitric oxide inhibitor," Neuroscience Letters, vol. 433, no. 1, pp. 59-64, 2008.

[41] Y.-L. Peng, Y.-N. Liu, L. Liu, X. Wang, C.-L. Jiang, and Y.$\mathrm{X}$. Wang, "Inducible nitric oxide synthase is involved in the modulation of depressive behaviors induced by unpredictable chronic mild stress," Journal of Neuroinflammation, vol. 9, article 75, 2012.

[42] A. L. López-López, H. J. Bonilla, M. D. C. Escobar Villanueva, M. P. Brianza, G. P. Vázquez, and F. J. A. Alarcón, "Chronic unpredictable mild stress generates oxidative stress and systemic inflammation in rats," Physiology and Behavior, vol. 161, pp. 15-23, 2016.

[43] X.-Y. Deng, J.-S. Xue, H.-Y. Li et al., "Geraniol produces antidepressant-like effects in a chronic unpredictable mild stress mice model," Physiology and Behavior, vol. 152, pp. 264271, 2015.

[44] Y. Yazir, T. Utkan, N. Gacar, and F. Aricioglu, "Resveratrol exerts anti-inflammatory and neuroprotective effects to prevent memory deficits in rats exposed to chronic unpredictable mild stress," Physiology \& Behavior, vol. 138, pp. 297-304, 2015.

[45] L. Chaieb, A. Antal, G. G. Ambrus, and W. Paulus, "Brainderived neurotrophic factor: Its impact upon neuroplasticity and neuroplasticity inducing transcranial brain stimulation protocols," Neurogenetics, vol. 15, no. 1, pp. 1-11, 2014.

[46] I. Branchi, N. Francia, and E. Alleva, "Epigenetic control of neurobehavioural plasticity: The role of neurotrophins," Behavioural Pharmacology, vol. 15, no. 5-6, pp. 353-362, 2004.

[47] E. Gumuslu, O. Mutlu, D. Sunnetci et al., "The antidepressant agomelatine improves memory deterioration and upregulates CREB and BDNF gene expression levels in unpredictable chronic mild stress (UCMS)-exposed mice," Drug Target Insights, vol. 8, pp. 11-21, 2014.

[48] W. Fu, H. Xie, M. Laudon, S. Zhou, S. Tian, and Y. You, "Piromelatine ameliorates memory deficits associated with chronic mild stress-induced anhedonia in rats," Psychopharmacology, vol. 233, no. 12, pp. 2229-2239, 2016. 


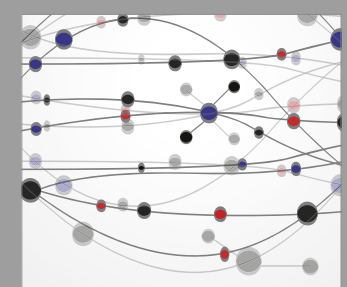

The Scientific World Journal
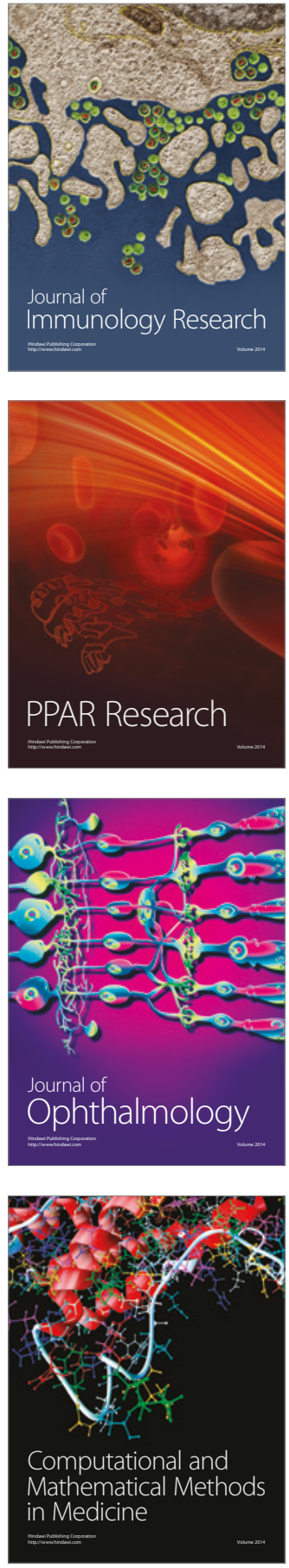

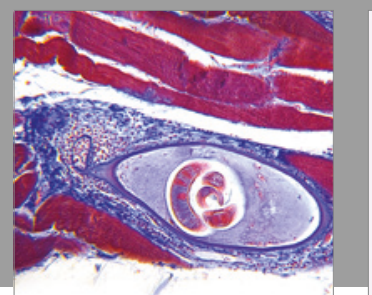

Gastroenterology Research and Practice
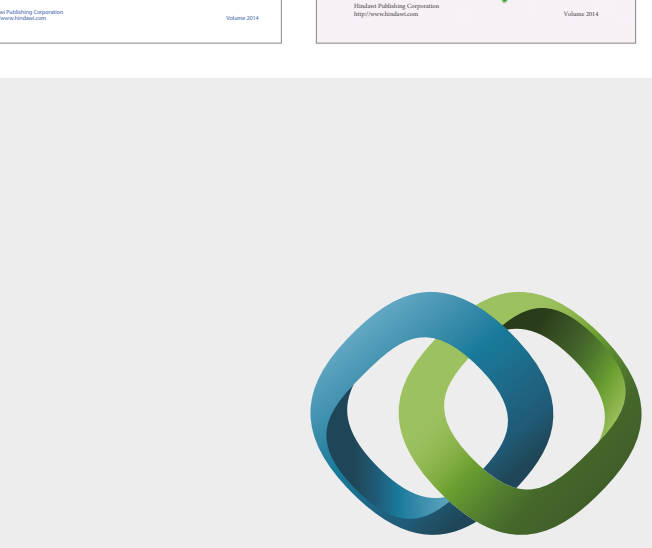

\section{Hindawi}

Submit your manuscripts at

https://www.hindawi.com
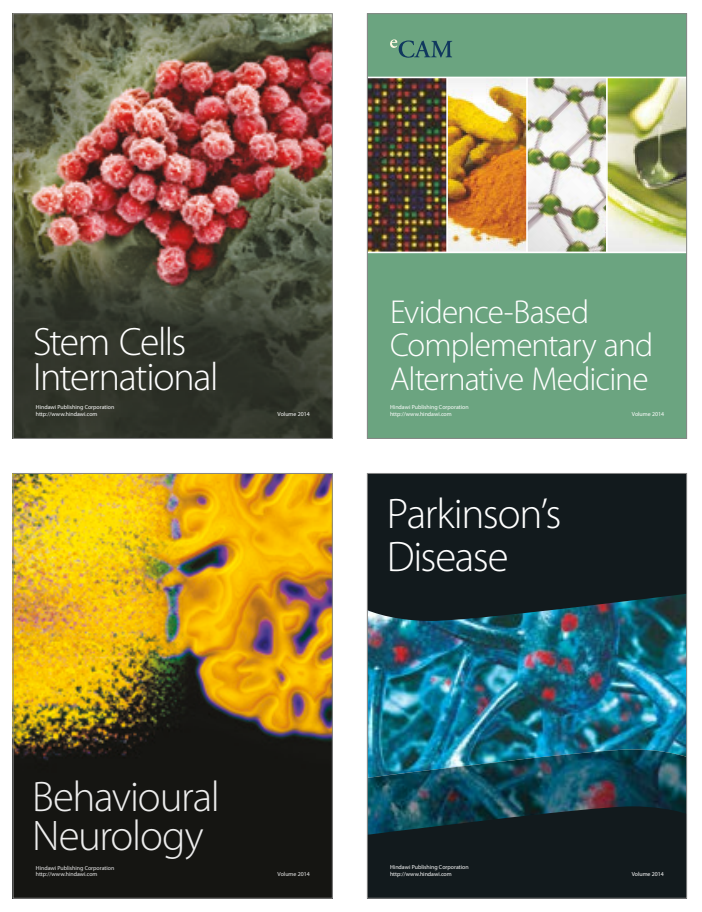
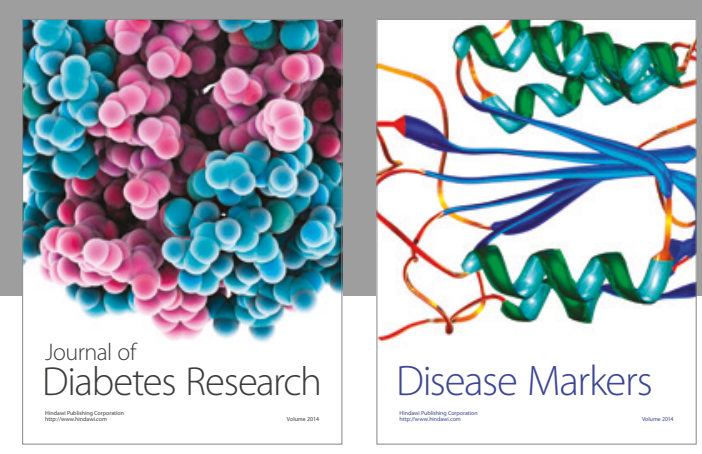

Disease Markers
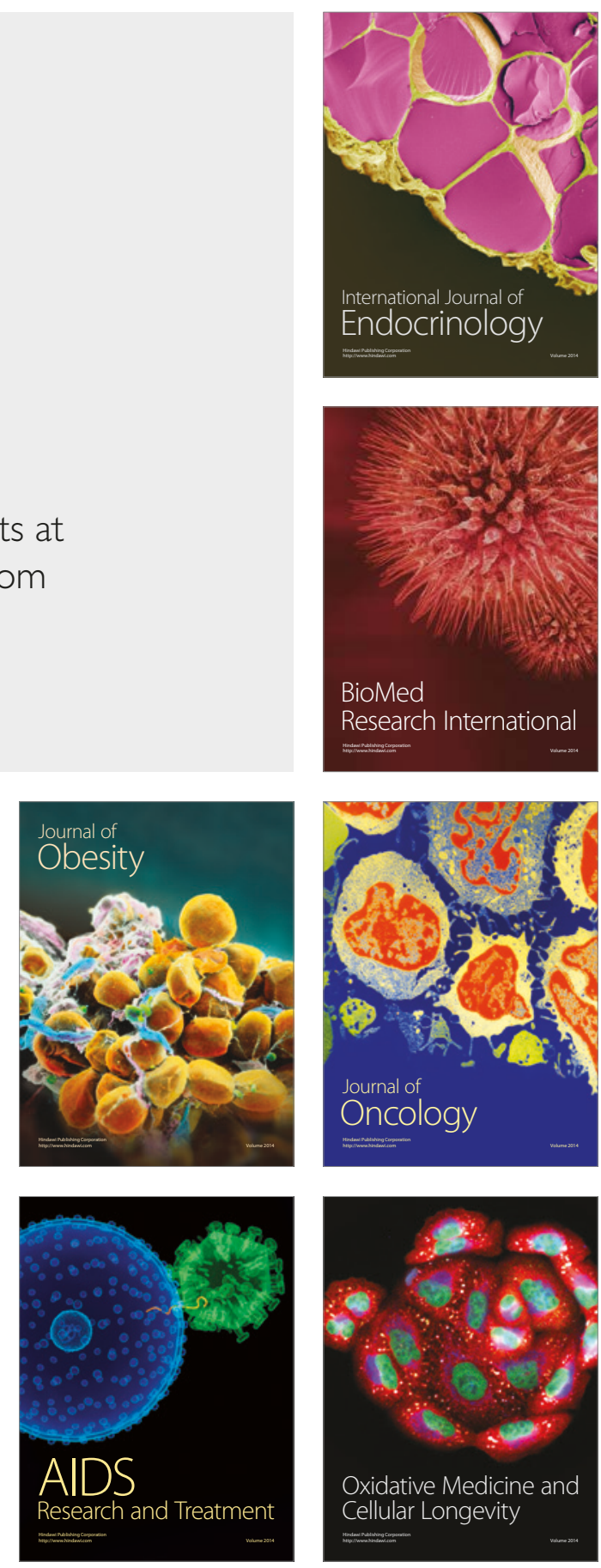